\title{
CLOSED INTEGRAL CURVES IN 3-SPACE AND ISOTOPIC TWO-DIMENSIONAL DEFORMATIONS
}

HERBERT SEIFERT

If there is given an isotopic deformation of a two-dimensional euclidean plane $E$ onto itself, one can adjoin to every point $P$ of $E$ a certain closed curve, the so-called "indicatrix" of $P(\$ 1)$. If the indicatrix does not pass through $P$, we introduce the order of $P$ relative to its indicatrix as the "rotation number" of the deformation at $P$. A relation between the rotation numbers in different points of $E(\S 3)$ and a formula for the rotation number in the general case of a "bounded deformation" ( $\$ 4)$ is established. This formula admits an application to the problem of closed integral curves of continuous vector fields in the 3-dimensional sphere.

1. Indicatrix and rotation number. An isotopic deformation of the plane $E$ is a set $\left\{A_{\lambda}\right\}(0 \leqq \lambda \leqq 1)$ of topological mappings of $E$ onto itself such that $A_{0}$ is the identical mapping and that $A_{\lambda}(P)$ depends continuously on $\lambda$ and $P$. The curve $A_{\lambda}(P)(0 \leqq \lambda \leqq 1)$ is called the trajectory of $P$. It is unessential that we confine $\lambda$ to the interval $0 \leqq \lambda \leqq 1$. Sometimes it will be advisable to take another closed interval $a \leqq \lambda \leqq b$.

Given the deformation $\left\{A_{\lambda}\right\}$ we can adjoin to every point $P$ of the plane $E$ an indicatrix, namely the curve

$$
A_{\lambda} A_{1} A_{\lambda}^{-1}(P) \quad(0 \leqq \lambda \leqq 1) .
$$

This curve is closed since (1) reduces to $A_{1}(P)$ both for $\lambda=0$ and $\lambda=1$. It may happen (for example, when the mappings $A_{\lambda}$ are commutative) that the indicatrix consists only of the point $A_{1}(P)$.

The point $P$ lies on its indicatrix if and only if for a suitable $\lambda$

$$
A_{\lambda} A_{1} A_{\lambda}^{-1}(P)=P
$$

or

$$
A_{1} A_{\lambda}^{-1}(P)=A_{\lambda}^{-1}(P) \text {. }
$$

Putting

$$
A_{\lambda}^{-1}(P)=Q
$$

we see from (2) that $Q$ is a fixed point of $A_{1}$ and from (3) that $P$ lies on the trajectory of $Q$. Thus the two statements " $P$ lies on its indi-

Received by the editors December 16, 1948. 
catrix" and " $P$ lies on the trajectory of a fixed point of $A_{1}$ " are equivalent.

If $P$ lies on its indicatrix, we call $P$ an exceptional point. Let $N$ be the set of all exceptional points. $N$ is empty if and only if $A_{1}$ is without fixed points. $N$ is closed. For if $P$ is a point of $E-N, P$ is not on its indicatrix. Then the same is true for all points of a sufficiently small neighborhood of $P$, as the indicatrix varies continuously with $P$. Thus all points of a sufficiently small neighborhood of $P$ belong to $E-N$, that is $N$ is closed.

Let $P$ be not an exceptional point. Then $P$ has a certain order relative to its indicatrix. The order of a point $P$ relative to a closed curve $q$ which does not pass through $P$ is defined to be $1 / 2 \pi$ times the total change of the angle between the vector ${ }^{1}\langle P Q\rangle$ and a fixed direction when $Q$ traverses the curve $q$. Of course the order depends on the orientation of the plane. We always consider a triangle $O A B$ positively oriented with $O$ being the origin, $A$ a point on the positive $x$-axis, $B$ a point on the positive $y$-axis. The order of $P$ relative to its indicatrix will be called the rotation number $\omega(P)$ of the deformation $\left\{A_{\lambda}\right\}$ at the point $P$. The plane $E$ is decomposed by the set $N$ of all exceptional points into a number of regions (components of $E-N$ ). If $P$ is allowed to vary continuously in a component of $E-N$, then the indicatrix of $P$ changes continuously and never passes through $P$. Hence the rotation number is constant on every component of $E-N$. In the special case that $N$ is the empty set or, which is the same, the mapping $A_{1}$ has no fixed points, the rotation number is constant throughout the plane. I.

EXAMPLES:

$$
A_{\lambda}: \quad x^{\prime}=x+\lambda, y^{\prime}=y .
$$

$A_{1}$ has no fixed points. The indicatrix of the point $(x, y)$ shrinks to the point $(x+1 ; y)$. The rotation number is equal to zero everywhere. II.

$$
\begin{aligned}
& A_{\lambda}: \quad x^{\prime}=x \cos 2 k \pi \lambda-y \sin 2 k \pi \lambda+\lambda \\
& y^{\prime}=x \sin 2 k \pi \lambda+y \cos 2 k \pi \lambda
\end{aligned}
$$

( $k$ an integer).

$A_{1}$ is the translation $x^{\prime}=x+1, y^{\prime}=y$. Therefore

$$
A_{\lambda} A_{1} A_{\lambda}^{-1}: \quad x^{\prime}=x \cos 2 k \pi \lambda, y^{\prime}=y+\sin 2 k \pi \lambda,
$$

the transform of a translation $A_{1}$ by a rotation $A_{\lambda}$ being again a trans-

\footnotetext{
${ }^{1}$ The vector from the initial point $P$ to the end point $Q$ will be designated by
} $\langle P Q\rangle$, the usual notation, an arrow over the points $P Q$, being difficult to print. 
lation. Thus the indicatrix of a point $P$ is the unit circle with center $P, k$ times traversed. The rotation number is equal to $k$ throughout the plane.

III.

$$
\begin{aligned}
& A_{\lambda}: \quad x^{\prime}=x \cos 2 k \pi \lambda-y \sin 2 k \pi \lambda, \\
& y^{\prime}=x \sin 2 k \pi \lambda+y \cos 2 k \pi \lambda
\end{aligned}
$$

( $k$ not an integer).

$A_{1}$ is the rotation around the origin through the angle $2 k \pi$. The origin is the only exceptional point. In all points the rotation number is defined and equal to zero because of the commutativity of the rotations (5).

2. Three-dimensional interpretation of the rotation numbers. We consider the plane $E$ in which the deformation $\left\{A_{\lambda}\right\}$ is given as the plane $z=0$ of an orthogonal $x y z$-coordinate system. Let $B_{\lambda}$ be the translation

$$
B_{\lambda}: \quad x^{\prime}=x, y^{\prime}=y, z^{\prime}=z+\lambda .
$$

Then $B_{\lambda} A_{\lambda}$ maps the plane $z=0$ onto the plane $z=\lambda$. For every $P \in E$ the points

$$
B_{\lambda} A_{\lambda}(P)=Q_{\lambda} \quad(0 \leqq \lambda \leqq 1)
$$

form a curve which lies in the three-dimensional set $Z$

$$
Z: \quad 0 \leqq z \leqq 1, \quad-\infty<x<+\infty,-\infty<y<+\infty
$$

and whose parameter $\lambda$ coincides with the $z$-coordinate. The orthogonal projection of the curve (7) into the plane $E$ is identical with the trajectory of $P$. (7) will be called the streamline of the point $P$. As the mapping $B_{\lambda} A_{\lambda}$ of the plane $z=0$ onto the plane $z=\lambda$ is one-to-one, there is exactly one streamline through every point of $Z$.

If one identifies every two points $(x, y, 0)$ and $(x, y, 1)$, one can continue on a streamline arbitrarily far: when one has arrived in a point $(x, y, 1)$, one continues on the streamline going out from $(x, y, 0)$. In particular one can start in an arbitrary point $Q_{\lambda}$ with $z$-coordinate $\lambda$ and traverse the streamline until $z$ has an increment of $\Delta z=1$. One arrives thus in a point $Q_{\lambda}^{\prime}$ in the plane $z=\lambda$. The mapping $Q_{\lambda} \rightarrow Q_{\lambda}^{\prime}$ of $Z$ onto itself will be called the return mapping $T$ of the deformation $\left\{A_{\lambda}\right\}$, the vector $\left\langle Q_{\lambda} Q_{\lambda}{ }^{\prime}\right\rangle$ which is attached to the point $Q_{\lambda}$ is termed the return vector of the point $Q_{\lambda}$. T is obviously characterized by the following properties:

(a) $T$ induces the mapping $A_{1}$ in the plane $z=0$.

(b) $T$ carries streamlines into streamlines.

(c) $T$ maps every plane $z=\lambda$ onto itself. 
In the plane $z=\lambda$ the mapping $T$ induces the mapping $A_{\lambda} A_{1} A_{\lambda}^{-1}$ provided that one pays attention to the transformation of the coordinates $x, y$ only. To prove this we introduce the points $P$ and $P^{\prime}$ in which the streamlines through $Q_{\lambda}$ and $Q_{\lambda}^{\prime}$ intersect the plane $z=0$. From (7) we have

$$
Q_{\lambda}=B_{\lambda} A_{\lambda}(P) ; \quad Q_{\lambda}^{\prime}=B_{\lambda} A_{\lambda}\left(P^{\prime}\right)
$$

and from property (a)

$$
P^{\prime}=A_{1}(P) .
$$

It follows that

$$
Q_{\lambda}^{\prime}=B_{\lambda} \cdot A_{\lambda} A_{1} A_{\lambda}^{-1} \cdot B_{\lambda}^{-1}\left(Q_{\lambda}\right),
$$

which was to be proved.

In particular $T$ induces in the plane $z=1$ (as well as in the plane $z=0$ ) the mapping $A_{1}$. Because of the properties (b) and (c), a point $Q$ of $Z$ remains fixed under the return mapping $T$ if and only if all points of the streamline through $Q$ remain fixed. So the fixed points of $T$ are the points of the streamlines passing through the fixed points of the mapping $A_{1}$ of the plane $z=0$. These streamlines will be termed the fixed lines of $T$. By orthogonal projection of the fixed lines onto $z=0$ one obtains exactly the set $N$ of exceptional points ( $\$ 1)$. Now we can give a 3-dimensional interpretation of the indicatrix and the rotation number $\omega(P)$ of a point $P$ in the plane $z=0$. Let $p$ be the segment $0 \leqq z \leqq 1$ through $P$ parallel to the z-axis, $T(p)$ the image of $p$ under the return mapping $T$, then the orthogonal projection of $T(p)$ onto the plane $z=0$ is the indicatrix of $P$.

This is an immediate consequence of the definition of the indicatrix and of the fact that $T$ induces the mapping $A_{\lambda} A_{1} A_{\lambda}^{-1}$ in the plane $z=\lambda$. So the rotation number $\omega(P)$ is up to the factor $2 \pi$ egual to the total change of the return vector when its initial point traverses the segment $p$.

3. Relation between the rotation numbers in different points. Henceforth we shall assume that the mapping $A_{1}$ which is defined in the plane $z=0$ only has isolated fixed points $F_{v}(\nu=1,2, \cdots)$. Let $\tilde{f}_{v}$ be the fixed line with the initial point $F_{\nu}$ oriented in the direction of increasing values of $z$. The end point of $\tilde{f}_{v}$ is the point of the plane $z=1$ which has the same $x y$-coordinates as $F_{r}$.

Every point $P$ of the three-dimensional domain $Z^{*}=Z-U \tilde{f}_{v}$ is the initial point of a return vector $\langle P Q\rangle$ which is different from zero. When $P$ traverses a singular 1 -simplex $s \subset Z^{*}$ there is a certain total 
change of the angle between $\langle P Q\rangle$ and the positive $x$-axis. This change, divided by $2 \pi$, is called the rotation number $\omega(s)$ of the 1 -simplex $s$. More generally we can adjoin to every 1-chain $\sigma=s_{1}$ $+\cdots+s_{k} \in Z^{*}$ a rotation number, namely $\omega(\sigma)=\omega\left(s_{1}\right)+\cdots$ $+\omega\left(s_{k}\right) . \omega(\sigma)$ is an integer if $\sigma$ is a cycle. As the return vector $\langle P Q\rangle$ depends continuously on $P$, there is for every $P \subset Z^{*}$ a neighborhood $U_{P}$ such that $\omega(\sigma)=0$ for every cycle in $U_{P}$. It follows that the rotation number is zero for every cycle in $Z^{*}$ which is homologous to zero in $Z^{*}$.

Let $k_{\nu}$ be a circle in the plane $z=0$ with center $F_{\nu}$ such that $F_{\nu}$ is the only fixed point of $A_{1}$ on the closed interior of $k_{\nu}$. Choose the orientation of $k_{\nu}$ so that the order of $F$, relative to $k_{\nu}$ is +1 . As the return vector in the plane $z=0$ runs from $P$ to $A_{1}(P)$ we find that $\omega\left(k_{v}\right)$ is the fixed point index $\gamma$, of $F$, under the mapping $A_{1}$ :

$$
\omega\left(k_{\nu}\right)=\gamma_{v} .
$$

The circles $k_{v}$ form a homology basis of dimension 1 of $Z^{*}$. For $Z^{*}$ is the topological product of $E-U F$, and the closed unit segment. Thus for an arbitrary 1-cycle $c \subset Z^{*}$ there is a homology

$$
c \sim \sum x_{\nu} k_{\nu} \text { on } Z^{*} \text {, }
$$

where of course only a finite number of coefficients $\chi_{\nu}$ are not $0 . \chi_{\nu}$ is obviously the intersection number of $\bar{f}_{v}$ and a 2 -chain $b \subset Z$ with boundary $c$ :

$$
\begin{aligned}
\chi_{v} & =S\left(\bar{f}_{v}, b\right), \\
\dot{b} & =c .
\end{aligned}
$$

The right side of (12) has a meaning provided that $b$ intersects neither the initial points nor the end points of the paths $\tilde{f}_{v}$. This always can be assumed. In formula (12) we make use of the orientation $\left(O, O_{1}, O_{2}, O_{3}\right)$ of $Z$ where $O, O_{1}, O_{2}, O_{3}$ are respectively the origin and points on the positive $x, y, z$-axis. With this orientation the intersection number of the oriented $z$-axis and the oriented $x y$-plane is +1 .

For the rotation number $\omega(c)$ we have from (10) and (11)

$$
\omega(c)=\sum \chi_{\nu} \gamma_{\nu} .
$$

Let $f_{\nu}$ be the orthogonal projection of $\tilde{f}_{v}$ into the plane $E(z=0)$, and $U, V$ two points of $E-U f_{p}$ whose rotation numbers we wish to compare. If $u$ and $v$ are the unit segments through $U$ and $V$ parallel to the $z$-axis, we have from the result of $\$ 2$

$$
\omega(U)=\omega(u), \quad \omega(V)=\omega(v) .
$$


We draw from $U$ to $V$ in the plane $z=0$ a path $q$ which avoids the other fixed points. By the translation $x^{\prime}=x, y^{\prime}=y, z^{\prime}=z+1, q$ is carried into the path $q_{1}$ in the plane $z=1$. Then

$$
c=q+v-q_{1}-u
$$

is a 1 -cycle in $Z^{*}$. Hence

$$
\omega(c)=\omega(v)-\omega(u)
$$

as the return vectors in corresponding points of $q$ and $q_{1}$ are equal. Let $b$ be the 2-chain that is described by $q$ under the translation into $q_{1}$. By the projection of $Z$ onto $E$ the intersection points of $b$ and $\tilde{f}_{v}$ are carried into the intersection points of $q$ and $f_{v}$. According to our choice of the orientations of $E$ and $Z$ the indices of the intersection points are not altered by the projection. So we have from (12)

$$
\chi_{\nu}=S\left(\tilde{f}_{v}, b\right)=S\left(f_{v}, q\right) .
$$

From (14), (15), (16), (17) we deduce the following theorem.

THEOREM 1. If $U$ and $V$ are not exceptional points relative to the deformation $\left\{A_{\lambda}\right\}$ and if the fixed points $F_{\nu}$ of $A_{1}$ have no limit point, then the rotation numbers $\omega(U)$ and $\omega(V)$ are connected by the relation

$$
\omega(V)-\omega(U)=\sum_{1}^{\infty} \gamma_{\nu} S\left(f_{v}, q\right),
$$

in which $\gamma_{\nu}$ is the fixed point index of $F_{\nu}$ under the mapping $A_{1}, f_{\nu}$ the trajectory of $F_{\nu}$ under the deformation $\left\{A_{\nu}\right\}$ and $S\left(f_{\nu}, q\right)$ the intersection number of $f_{2}$ and a curve $q$ running from $U$ to $V$.

In the formulation of the theorem the condition that $q$ should avoid the fixed points $F$, is obviously unnecessary.

4. Bounded deformations. An isotopic deformation $\left\{A_{\lambda}\right\}$ of the plane is called bounded if there is an $\epsilon>0$ such that the diameter of the trajectory of every point is less than $\epsilon$. More precisely $\left\{A_{\lambda}\right\}$ is termed an $\epsilon$-deformation.

We shall generalize this concept in so far as we shall only assume that the deformation $\left\{A_{\lambda}\right\}(0 \leqq \lambda \leqq 1)$ is defined in a certain domain $G$ of the plane $E$ (not necessarily in the whole plane). In other words we shall assume that (a) $A_{\lambda}(0 \leqq \lambda \leqq 1)$ is a topological mapping of $G$ into the plane $E$ (not necessarily onto $G$ ); (b) $A_{\lambda}$ depends continuously on $P$ and $\lambda$; (c) $A_{0}$ is the identity mapping; (d) for every point $P \in G$ the diameter of the trajectory of $P$ under the deformation is less than $\epsilon$. Then $\left\{A_{\lambda}\right\}$ is called an isotopic $\epsilon$-deformation of $G$. If for 
some $P \in G$ the curve $A_{\lambda} A_{1} A_{\lambda}^{-1}(P)(0 \leqq \lambda \leqq 1)$ exists, it is called the indicatrix of $P$. If $P$ does not lie on its indicatrix, the order of $P$ relative to its indicatrix is called the rotation number of the deformation at $P$. These definitions obviously agree with those of $\$ 1$.

Let in particular $G$ be the square

$$
\left|x-x_{0}\right|<a, \quad\left|y-y_{0}\right|<a \quad(a>0)
$$

and

$$
\epsilon<a / 2 \text {. }
$$

Then the squares

$$
\begin{array}{ll}
\left|x-x_{0}\right|<a-\epsilon, & \left|y-y_{0}\right|<a-\epsilon, \\
\left|x-x_{0}\right|<a-2 \epsilon, & \left|y-y_{0}\right|<a-2 \epsilon
\end{array}
$$

exist. $A_{\lambda}$ maps (19) onto a point set which contains (20). So $A_{\lambda}^{-1}$ is defined on (20). Similarly, $A_{\lambda} A_{1} A_{\lambda}^{-1}$ is defined on (21). For $A_{\lambda}^{-1}$ maps (21) into (20); $A_{1}$ maps (20) into (19), and $A_{\lambda}$ is defined everywhere on (19).

LEMMA 1. If $\left\{A_{\lambda}\right\}(0 \leqq \lambda \leqq 1)$ is an $\epsilon$-deformation of the square (19) such that

$$
\epsilon<a / 3
$$

and for every $P$ of (19)

$$
x(P)<x\left(A_{1}(P)\right),
$$

$x(P)$ being the abscissa of $P$, then in all points of the square (21) the rotation number is defined and equal to zero.

Proof. We have shown that for every point $P$ of (19) the indicatrix $A_{\lambda} A_{1} A_{\lambda}^{-1}(P)$ is defined. It does not pass through $P$ because otherwise for a suitable $\lambda, A_{\lambda}^{-1}(P)$ would be a fixed point of $A_{1}$, contrary to (23). Hence in all points of (21) the rotation number is defined and consequently constant. So it will be sufficient to calculate it for a single point, say for

$$
Q=\left(x_{0}-\epsilon, 0\right)
$$

Let

$$
\bar{Q}=\left(x_{0}+\epsilon, 0\right) \text { and } Q^{\prime}=A_{1}(Q) .
$$

From (23) and the fact that $Q$ and $Q^{\prime}$ have a distance less than $\epsilon$, it follows that 


$$
x(Q)<x\left(Q^{\prime}\right)<0 .
$$

Let $R_{\mu}$ be the point that divides the segment $Q^{\prime} \bar{Q}$ in the ratio $\mu$ : $(1-\mu)(0 \leqq \mu \leqq 1)$. We consider the vector

$$
v(\lambda, \mu)=\left\{\begin{array}{lr}
\left\langle A_{\lambda}(Q) A_{\lambda}\left(R_{\mu}\right)\right\rangle & \text { for } 0 \leqq \lambda \leqq 1,0 \leqq \mu \leqq 1, \\
\left\langle A_{\lambda(1+\mu)}(Q) A_{\lambda} A_{1} A_{\lambda}^{-1} A_{\lambda(1+\mu)}(Q)\right\rangle & \text { for } 0 \leqq \lambda \leqq 1,-1 \leqq \mu \leqq 0 .
\end{array}\right.
$$

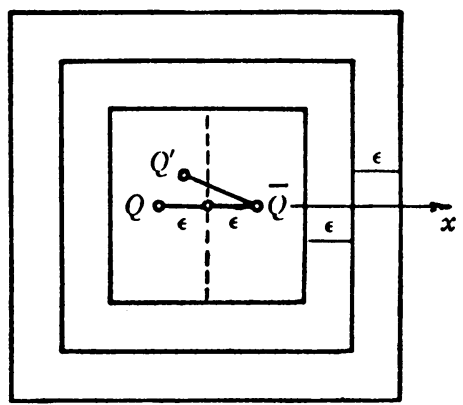

FIG. 1

The two definitions coincide for $\mu=0$ since $R_{0}=Q^{\prime}=A_{1}(Q)$ and $A_{\lambda(1+0)}=A_{\lambda} . \quad v(\lambda, \mu)$ vanishes nowhere in the rectangle

$$
0 \leqq \lambda \leqq 1, \quad-1 \leqq \mu \leqq 1 .
$$

In fact, from (24) and

$$
x\left(Q^{\prime}\right) \leqq x\left(R_{\mu}\right),
$$

it follows that $Q$ and $R_{\mu}$ are different. So the same is true for $A_{\lambda}(Q)$ and $A_{\lambda}\left(R_{\mu}\right)$, that is, $v(\lambda, \mu) \neq 0$ for $0 \leqq \lambda \leqq 1,0 \leqq \mu \leqq 1$. Similarly, for $-1 \leqq \mu \leqq 0$ the point $S=A_{\lambda}^{-1} A_{\lambda(1+\mu)}(Q)$ lies within the circle of $Q$ with radius $2 \epsilon, A_{\lambda}^{-1}$ and $A_{\lambda(1+\mu)}$ being $\epsilon$-mappings. From (22) and (23) we deduce that $A_{1}(S)$ is contained in (19) and

$$
S \neq A_{1}(S) \text {. }
$$

It follows by multiplication with $A_{\lambda}$

$$
A_{\lambda}(S) \neq A_{\lambda} A_{1}(S) \text {, }
$$

that is, $v(\lambda, \mu) \neq 0$ for $0 \leqq \lambda \leqq 1 ;-1 \leqq \mu \leqq 0$. Next one can verify that on the edges $\lambda=0, \lambda=1, \mu=1$ of the rectangle (26) the end point of the vector $v(\lambda, \mu)$ always has greater abscissa than the initial point. Indeed we have for $\lambda=0$ 


$$
v(0, \mu)=\left\{\begin{array}{l}
\left\langle Q R_{\mu}\right\rangle \\
\left\langle Q Q^{\prime}\right\rangle
\end{array}\right.
$$

$$
\begin{array}{r}
(0 \leqq \mu \leqq 1), \\
(-1 \leqq \mu \leqq 0)
\end{array}
$$

and

$$
x(Q)<x\left(Q^{\prime}\right) \leqq x\left(R_{\mu}\right)
$$

from (24) and (27). Similarly for $\lambda=1$

$$
v(1, \mu)=\left\{\begin{array}{lr}
\left\langle Q^{\prime} A_{1}\left(R_{\mu}\right)\right\rangle & (0 \leqq \mu \leqq 1), \\
\left\langle A_{1+\mu}(Q) A_{1} A_{1+\mu}(Q)\right\rangle & (-1 \leqq \mu \leqq 0)
\end{array}\right.
$$

and

$$
\begin{aligned}
x\left(Q^{\prime}\right) \leqq x\left(R_{\mu}\right) & <x\left(A_{1}\left(R_{\mu}\right)\right), \\
x\left(A_{1+\mu}(Q)\right) & <x\left(A_{1} A_{1+\mu}(Q)\right)
\end{aligned}
$$

from (27) and (23). Finally for $\mu=1$

$$
v(\lambda, 1)=\left\langle A_{\lambda}(Q) A_{\lambda}(\bar{Q})\right\rangle
$$

and

$$
x\left(A_{\lambda}(Q)\right)<0, \quad x\left(A_{\lambda}(\bar{Q})\right)>0
$$

since $x(Q)=x_{0}-\epsilon, x(\bar{Q})=x_{0}+\epsilon$ and $A_{\lambda}$ is an $\epsilon$-mapping.

Now we consider $v(\lambda, \mu)$ for $\mu=-1$. We have

$$
v(\lambda,-1)=\left\langle Q A_{\lambda} A_{1} A_{\lambda}^{-1}(Q)\right\rangle .
$$

This is the vector that leads from $Q$ to the point $(\lambda)$ on the indicatrix of $Q$. So the rotation number $\omega(Q)$ is given (up to a factor $2 \pi$ ) by the total change of the angle between the positive $x$-axis and the vector $v(\lambda,-1)$ when $\lambda$ goes from 0 to 1 . Since $v(\lambda, \mu)$ does not vanish in the rectangle (26) we come to the same result when we continue $v(\lambda, \mu)$ along the three other edges of the rectangle, but here the end point of $v(\lambda, \mu)$ has always greater abscissa than the initial point. Therefore

$$
\omega(Q)=0,
$$

q.e.d.

THEOREM 2. Let $\left\{A_{\lambda}\right\}(0 \leqq \lambda \leqq 1)$ be a bounded isotopic deformation of the plane $E$, such that $A_{1}$ has at most isolated fixed points $F_{1}, F_{2}, \cdots$. If $f_{v}$ is the trajectory of $F_{v}$, then for a point $V$ which is on none of the $f_{v}$ the rotation number $\omega(V)$ is given by

$$
\omega(V)=\sum_{1}^{\infty} \gamma_{0} \theta_{v}
$$


where $\theta_{\nu}$ is the order of $V$ relative to $f_{\nu}$ and $\gamma_{\nu}$ is the fixed point index of $F_{\nu}$ at the mapping $A_{1}$.

An immediate consequence of Theorem 2 is the following:

Theorem 3. If $\left\{A_{\lambda}\right\}(0 \leqq \lambda \leqq 1)$ is a bounded isotopic deformation of the plane and $A_{1}$ is without fixed points, then the rotation number of the deformation is zero in every point of the plane.

Proof of theorem 2. Because of the boundedness of the deformation there is a $\delta>0$ so that for every point the diameter of the trajectory is less than $\delta$. We choose the coordinate system so that $V$ gets the coordinates $x=-4 \delta, y=0$.

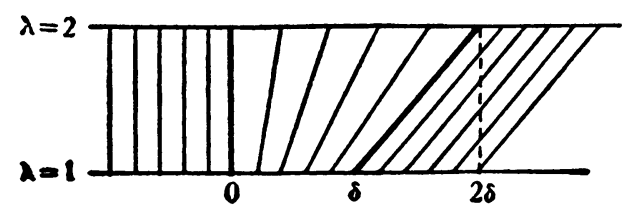

FIG. 2

We extend the deformation $\left\{A_{\lambda}\right\}(0 \leqq \lambda \leqq 1)$ to a deformation whose parameter $\lambda$ runs from 0 to 3 by the following definition: For $1 \leqq \lambda \leqq 2$ let $\left\{A_{\lambda} A_{1}^{-1}\right\}$ be the $\delta$-deformation

$$
\begin{array}{llr}
x^{\prime}=x, & y^{\prime}=y & (x \leqq 0), \\
x^{\prime}=\lambda x, & y^{\prime}=y & (0 \leqq x \leqq \delta), \\
x^{\prime}=x+(\lambda-1) \delta, & y^{\prime}=y & (x \geqq \delta)
\end{array}
$$

whose streamlines are shown by Fig. 2 .

For $2 \leqq \lambda \leqq 3$ let $\left\{A_{\lambda} A_{2}^{-1}\right\}$ be an isotopic $\eta$-deformation $(\eta<\delta)$ and $A_{3}$ a (necessarily topological) simplicial mapping of the plane with only isolated fixed points. ${ }^{2}$ In the following we shall restrict $\eta$ by some further conditions.

The deformation $\left\{A_{\lambda}\right\}(0 \leqq \lambda \leqq 3)$ is denoted by $A_{(0,3)}$ and the meaning of $A_{(0,1)}$ and $A_{(0,2)}$ is similar. $A_{(0,1)}, A_{(0,2)}, A_{(0,3)}$ is respectively a $\delta$-, $2 \delta$-, $3 \delta$-deformation. All quantities (fixpoints, rotation numbers, trajectories) concerning $A_{(0,2)}\left(A_{(0,3)}\right)$ will be distinguished from the corresponding quantities of $A_{(0,1)}$ by a prime (two

${ }_{2}$ Every typological mapping $A_{2}$ of the plane onto itself can be carried into a simplicial mapping by an isotopic $\eta$-deformation ( $\eta$ arbitrarily small). (See Graeub, Semilineare Abbildungen, to appear in Sitzungsberichte der Heidelberger Akademie der Wissenschaften. This deformation may be omitted if $A_{2}$ has only isolated fixed points. 
primes). For example, the fixed points of $A_{3}$ will be designated by $F_{\nu}^{\prime \prime}$, and $f_{v}^{\prime \prime}$ is the trajectory of $F_{\nu}^{\prime \prime}$ under $A_{(0,3)}$.

We consider these deformations

(a) in the left half-plane $L: x<0$,

(b) in the right half-plane $R: x>2 \delta$,

(c) in the middle area $M: 0 \leqq x \leqq 2 \delta$.

The fixed points $F_{v}$ of $A_{1}$ are denoted by $F_{l_{p}}, F_{r_{p}}$, or $F_{m_{p}}$, according as they belong to $L, R$, or $M$. The fixed points $F_{\eta}^{\prime}$ of $A_{2}$ and $F_{v}^{\prime \prime}$ of $A_{\mathbf{3}}$ will be classified and denoted in a similar way.

If $P$ is a point of $R$, we have $x(P)-x\left(A_{1}(P)\right)<\delta$ since $A_{(0,1)}$ is a $\delta$-deformation; moreover $x\left(A_{2}(P)\right)-x\left(A_{1}(P)\right)=\delta$ because of (29), therefore

$$
x(P)<x\left(A_{2}(P)\right) .
$$

It follows that for all points $P$ of a bounded closed subset of $R$ and for sufficiently small $\eta$ the inequality

$$
x(P)<x\left(A_{3}(P)\right)
$$

holds, for example for the points of the square

$$
|x-13 \delta| \leqq 10 \delta ; \quad|y| \leqq 10 \delta .
$$

Since $A_{(0,3)}$ is a $3 \delta$-deformation, we have for the rotation number $\omega^{\prime \prime}(U)$ at the center $U=(13 \delta, 0)$ of the square (32) because of Lemma 1

$$
\omega^{\prime \prime}(U)=0 .
$$

At the point $V=(-4 \delta, 0)$ the rotation number $\omega(V)$ relative to the deformation $A_{(0,1)}$ is defined by hypothesis, that is, the indicatrix $A_{\lambda} A_{1} A_{\lambda}^{-1}(V)(0 \leqq \lambda \leqq 1)$ does not pass through $V$. So the same is true for the indicatrix $A_{\lambda} A_{3} A_{\lambda}^{-1}(V)(0 \leqq \lambda \leqq 3)$ relative to the deformation $A_{(0,3)}$, provided that $\eta$ is sufficiently small. For under this condition the two indicatrices will differ arbitrarily little. So $\omega^{\prime \prime}(V)$ is defined and equal to $\omega(V)$ :

$$
\omega^{\prime \prime}(V)=\omega(V) .
$$

Now we can apply Theorem 1 on the deformation $A_{(0,3)}$ and the points $U, V$ :

$$
\omega(V)=\omega^{\prime \prime}(V)=\omega^{\prime \prime}(U)+\sum \gamma_{\nu}^{\prime \prime} S\left(f_{\nu}^{\prime \prime}, q\right) .
$$

$q$ may be chosen to be the straight segment $U V$. (33) and (35) imply

$$
\omega(V)=\sum \gamma_{l_{p}}^{\prime \prime} S\left(f_{l_{p}}^{\prime \prime}, q\right)+\sum \gamma_{m_{\nu}}^{\prime \prime} S\left(f_{m_{\nu}}^{\prime \prime}, q\right)+\sum \gamma_{r_{p}}^{\prime \prime} S\left(f_{r_{p}, q}^{\prime \prime}, q .\right.
$$


Since $A_{(0,3)}$ is a $3 \delta$-deformation and there are no fixed points of $A_{3}$ in the square (32) because of (31), we have $S\left(f_{r_{y}}^{\prime \prime}, q\right)=0$ so that the third term in (36) vanishes. But also the second term on the right side of (36) is zero. For $f_{m_{p}}^{\prime \prime}$, being a trajectory of a point of $M$, lies in the strip $-3 \delta<x<5 \delta$ and consequently has with $q$ the intersection number 0 . Since the trajectory $f_{l y}^{\prime \prime}$ is in the half-plane $x<3 \delta$, the intersection number $S\left(f_{i v}^{\prime \prime}, q\right)$ is not altered when we replace $q$ by the ray drawn from $V$ in the direction of the positive $x$-axis. In other words, $S\left(f_{v}^{\prime \prime}, q\right)$ is equal to the order $\theta_{l_{v}}^{\prime \prime}$ of $V$ relative to $f_{v}^{\prime \prime}$. So (36) implies

$$
\omega(V)=\sum \gamma_{l_{v}}^{\prime \prime} \theta_{l_{\nu}}^{\prime \prime}
$$

On the other hand we consider expression (28): $\sum \gamma_{\nu} \theta_{\nu}$. Let $K$ be the closed circular disk with center $V$ whose radius $\rho$ is chosen so that $3 \delta<\rho<4 \delta$ and that no fixed point of $A_{1}$ is on the boundary of $K$. If $F_{\nu}$ is a fixed point of $A_{1}$ which does not lie on $K$, the trajectory

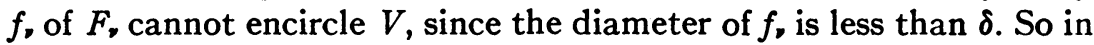
(28) we have only to consider the fixed points on $K$. Let these be $F_{1}, F_{2}, \cdots, F_{p}$. We choose $\beta>0$ so small that the open $\beta$-neighborhoods $U_{\beta}\left(F_{v}\right)$ for $\nu=1,2, \cdots, p$ belong to $K$ and do not intersect one another. Since $A_{1}$ has no fixed point on the closed set

$$
K-\bigcup_{v=1}^{p} U_{\beta}\left(F_{v}\right)=H,
$$

there is an $\alpha>0$ such that for every $P \in H$ the distance of $P$ and $A_{1}(P)$ is not less than $\alpha$. If we choose $\eta<\alpha$, then also $A_{3}$ has no fixed points on $H$ and we have

$$
\sum_{i=1}^{h_{r}} \gamma_{v, i}^{\prime \prime}=\gamma_{v}
$$

if $F_{v, 1}^{\prime \prime}, F_{v, 2}^{\prime \prime}, \cdots, F_{v, h_{\nu}}^{\prime \prime}$ are the fixed points of $A_{z}$ on $U_{\beta}\left(F_{\eta}\right)$ and $\boldsymbol{\gamma}_{\nu, 1}^{\prime \prime}, \boldsymbol{\gamma}_{\nu, 2}^{\prime \prime}, \cdots, \boldsymbol{\gamma}_{\nu, h}^{\prime \prime}$, are their indices. Moreover, the trajectory of $F_{v, i}^{\prime \prime}$ under the deformation $A_{(0,3)}$ differs arbitrarily little from the path of $F_{i}$ under $A_{(0,1)}$ (provided that $\eta$ is sufficiently small). Therefore we may assume that the orders $\theta_{\nu, i}^{\prime \prime}$ and $\theta_{\nu}$ of $V$ relative to these trajectories are equal:

$$
\theta_{v, i}^{\prime \prime}=\theta_{v}
$$

So we obtain

$$
\sum_{\nu=1}^{\infty} \gamma_{\nu} \theta_{\nu}=\sum_{\nu=1}^{p} \gamma_{\nu} \theta_{\nu}=\sum_{\nu=1}^{p} \sum_{i=1}^{h_{\nu}} \gamma_{p, i}^{\prime \prime} \theta_{\nu, i}^{\prime \prime} .
$$


The right sides of (37) and (40) differ only by terms $\gamma_{l_{v}}^{\prime \prime} \theta_{l_{v}}^{\prime \prime}$ that correspond to fixed points $F_{l v}^{\prime \prime}$ not on $K$. But these have a distance $>\rho>3 \delta$ from $V$, such that the corresponding trajectories $f_{i_{\nu}}^{\prime \prime}$, being less than $3 \delta$ in diameter, cannot encircle $V$. So we have $\theta_{b v}^{\prime \prime}=0$ for these $F_{l,}^{\prime \prime}$. It follows that the right sides of (37) and (40) are equal, hence

$$
\omega(V)=\sum_{\nu=0}^{\infty} \gamma_{\nu} \theta_{\nu}
$$

q.e.d.

Theorems 1 and 2 admit generalizations for the case where the deformations $\left\{A_{\lambda}\right\}(0 \leqq \lambda \leqq 1)$ are only defined in a certain open region $G$, not necessarily in the whole plane.

THeOREM $1^{\prime}$. Let $U$ and $V$ be two points of a plane open region $G$. Then there exists an $\epsilon>0$ with the following property: Formula (18) holds for every isotopic $\epsilon$-deformation $\left\{A_{\lambda}\right\}(0 \leqq \lambda \leqq 1)$ of $G$ for which $A_{1}$ has at most isolated fixed points (provided that the rotation numbers $\omega(U)$ and $\omega(V)$ are defined). $q \subset G$ is a path leading from $U$ to $V$.

THEOREM 2'. Let $V$ be a point of a plane open region $G$. Then there is an $\epsilon>0$ with the following property: Formula (28) holds for every isotopic $\epsilon$-deformation $\left\{A_{\lambda}\right\}(0 \leqq \lambda \leqq 1)$ of $G$ for which $A_{1}$ has at most isolated fixed points (provided that the rotation number $\omega(V)$ is defined).

THEOREM 3'. Let $V$ be a point of a plane open region $G$. Then there is an $\epsilon>0$, such that $\omega(V)=0$ for every isotopic $\epsilon$-deformation of $G$ for which $A_{1}$ is without fixed points.

Theorem $3^{\prime}$ is contained in Theorem $2^{\prime}$. The proofs of Theorems $1^{\prime}$ and $2^{\prime}$ differ only unessentially from those of Theorems 1 and 2. One only has to choose $\epsilon$ so small that $V$ respectively $U$ and $V$ are sufficiently far from the boundary of $G$ (compared with $\epsilon$ ) in order that the constructions made in the proofs of Theorems 1 and 2 become possible. In the proof of Theorem $2^{\prime}$ use has to be made of the fact that every homeomorphic mapping of $G$ into the plane can be deformed by an isotopic $\eta$-deformation ( $\eta$ arbitrarily small) into a simplicial mapping with only isolated fixed points.

5. Closed integral curves of vector fields in the three-dimensional sphere. If at every point of a differentiable $n$-dimensional manifold there is given a nonvanishing tangent vector which varies continuously with that point we speak of a continuous vector field. The necessary and sufficient condition for the existence of a continuous 
vector field in a closed manifold is the vanishing of the Euler characteristic. A differentiable curve which at each of its points is tangent to the field vector at that point is called an integral curve of the field. It is unknown if every continuous vector field of the three-dimensional sphere $S^{3}$ contains a closed integral curve. In the following we consider on $S^{3}$ a special class of vector fields for which the existence of a closed integral curve is a consequence of Theorem $3^{\prime}$.

Let $S^{3}$ be the three-dimensional unit-sphere of the four-dimensional euclidean space

$$
x_{1}^{2}+x_{2}^{2}+x_{3}^{2}+x_{4}^{2}=1 .
$$

We consider on $S^{3}$ the field $C$ of the "Clifford-parallel" vectors

$$
d x_{1}=-x_{2}, \quad d x_{2}=x_{1}, \quad d x_{3}=-x_{4}, \quad d x_{4}=x_{3},
$$

whose integral curves are great circles. They will be called the Clifford-circles of $S^{3}$. We choose the orientation of $S^{3}$ so that two different Clifford-circles (each of which has a definite orientation by (42)) have the looping coefficient +1 .

Let $\widetilde{C}$ be a continuous vector field on $S^{3}$ which differs sufficiently little from $C$, that is, the angle between the vector of $C$ and that of $\widetilde{C}$ is at every point of $S^{3}$ smaller than $\alpha$ ( $\alpha$ sufficiently small). Moreover, we suppose that through every point of $S^{3}$ there passes exactly one integral curve. We shall prove that for sufficiently small $\alpha$ there exists at least one closed integral curve in $\tilde{C}$.

The integral curves of $\tilde{C}$ will be called the streamlines of $S^{3}$. Let $\beta$ be a fixed number between 0 and $\pi / 2, P$ a point on $S^{3}, c$ the Clifford circle through $P, U_{\beta}(c)$ the (open) $\beta$-neighborhood of $c$. We introduce in $U_{\beta}(c)$ coordinates $x, y, z: P$ is the origin of the coordinate system, $x=$ const., $y=$ const. are the Clifford-circles in $U_{\beta}(c), z=$ const. are the two-dimensional great spheres orthogonal to $c ; x$ and $y$ are Riemann normal coordinates on $z=0$ and $z$ is the arclength on $c$, divided by $2 \pi$. There is a one-to-one correspondence between the points of $U_{\beta}(c)$ and the points of the set

$$
x^{2}+y^{2}<\beta^{2}, \quad 0 \leqq z<1,
$$

of euclidean $x y z$-space. The streamline which passes through $P$ admits the representation

$$
x=\phi(z), \quad y=\psi(z) .
$$

$\phi(z), \psi(z)$ are continuous functions of $z$ and defined for $0 \leqq z \leqq 1$ if $\alpha$ was chosen sufficiently small. Let $D(P)$ be the point $x=\phi(1)$, $y=\psi(1), z=1$ of $S^{z}$. Since $P$ was an arbitrary point the representation 
$P \rightarrow D(P)$ is defined everywhere on $S^{3}$. It can be considered as the final result of an isotopic deformation along the streamlines and therefore is a homeomorphic mapping with degree +1 . We have to prove that $D$ has fixed points.

For this purpose we choose once for all a Clifford-circle $c^{*}$ and consider besides $D$ a mapping $T$ which is defined only in the $\beta / 2$-neighborhood $U_{\beta / 2}\left(c^{*}\right)$ of $c^{*}$. As previously the points of $U_{\beta}\left(c^{*}\right)$ are referred to coordinates $x, y, z$. Let $Q\left(x_{0}, y_{0}, z_{0}\right)$ be a point of $U_{\beta / 2}\left(c^{*}\right)$ and

$$
\begin{aligned}
x=f\left(x_{0}, y_{0}, z_{0}, \lambda\right), y=g\left(x_{0}, y_{0}, z_{0}, \lambda\right), z & =z_{0}+\lambda \\
(0 \leqq \lambda & \left.\leqq 1, x^{2}+y^{2}<\beta^{2}\right)
\end{aligned}
$$

the streamline passing through $Q\left(x_{0}, y_{0}, z_{0}\right)$. For the existence of the representation (43) we must again suppose $\alpha$ to be small enough.

Let $T(Q)$ be the point

$$
x=f\left(x_{0}, y_{0}, z_{0}, 1\right), y=g\left(x_{0}, y_{0}, z_{0}, 1\right), z=z_{0}+1 .
$$

In general the mappings $Q \rightarrow T(Q)$ and $Q \rightarrow D(Q)$ do not coincide on $U_{\beta / 2}\left(c^{*}\right)$, for $D(Q)$ is on the great sphere which passes through $Q$ and which is orthogonal to the Clifford-circle through $Q$, while $T(Q)$ is on the great sphere which passes through $Q$ and which is orthogonal to the Clifford-circle $c^{*}$. However, if $Q$ is on $c^{*}$, we have $D(Q)=T(Q)$ and consequently

$$
T\left(c^{*}\right)=D\left(c^{*}\right) .
$$

(43) defines for $z_{0}=0$ an isotopic deformation

$$
x=f\left(x_{0}, y_{0}, 0, \lambda\right), \quad y=g\left(x_{0}, y_{0}, 0, \lambda\right) \quad(0 \leqq \lambda \leqq 1)
$$

of the two-dimensional region $x_{0}^{2}+y_{0}^{2}<\beta^{2} / 4$.

In order to prove our theorem indirectly we shall suppose that there is no closed streamline. Then the final mapping $(\lambda=1)$ of $(45)$ is without fixed points so that for sufficiently small $\alpha$ we may apply Theorem $3^{\prime}$. This means that the point $\left(x_{0}=0, y_{0}=0\right)$ has the order 0 relative to its indicatrix. Now, the indicatrix is the projection of $T\left(c^{*}\right)$ along the Clifford-circles, and since every two Clifford-circles have the looping coefficients +1 , it follows that $c^{*}$ and $T\left(c^{*}\right)$ have the looping coefficient +1 . Therefore by (44)

$$
\text { looping coefficient }\left(c^{*}, D\left(c^{*}\right)\right)=+1 \text {. }
$$

Every two Clifford-circles have a definite spherical distance. If the distance is $\pi / 2$ the Clifford-circles are called conjugate. In 4-dimensional $x_{1}, \cdots, x_{4}$-space the planes of conjugate Clifford-circles are orthogonal. We deform the mapping $D$ of $S^{3}$ into a mapping $D_{1}$ which 
maps every Clifford-circle into its conjugate. We only have to move $D(P)$ on the arc $P D(P)$ into the point $D_{1}(P)$ whose distance from $P$ is $\pi / 2$. Therefore we have from (46)

$$
\text { looping coefficient }\left(c^{*}, D_{1}\left(c^{*}\right)\right)=+1 \text {. }
$$

This means that $D_{1}$ maps every Clifford-circle with degree +1 onto its conjugate. On the other hand one can consider the set of the Clifford-circles as a fibre bundle whose base space is a two-sphere $S^{2} . D_{1}$ induces in $S^{2}$ a one-to-one mapping without fixed points which consequently has the degree -1 . This together with the fact that the fibres are mapped with degree +1 implies that $D_{1}$ and $D$ have degree -1 in contradiction to our previous statement that the degree of $D$ is +1 . This proves the following theorem:

THEOREM 4. A continuous vector field on the 3-sphere which differs sufficiently little from a field of Clifford-parallels and which sends through every point exactly one integral curve has at least one closed integral-curve.

This result can be generalized. If in our previous notation $P=D(P)$, we say that the streamline through $P$ closes after one circulation. Let $c^{*}$ be the Clifford-circle through $P$ and $T$ the above-mentioned mapping of the $\beta / 2$ neighborhood of $c^{*}$. Then $T$ induces in the surface $z=$ const. that contains $P$ a mapping with the fixed point $P$. If $P$ is an isolated fixed point we may assign its index to the closed streamline through $P$. Suppose that there is only a finite number of streamlines which close after one circulation. Then one can prove by means of Theorem 2' that the sum of the indices of these streamlines is always equal to 2 . More generally, if $S^{3}$ is replaced by an arbitrary three-dimensional fibre-bundle, this sum is equal to the Euler characteristic of the two-dimensional base space.

UNIVERSITY OF HEIDELBERG 